\title{
Prévision opérationnelle des apports de la Durance à Serre-Ponçon à l'aide du modèle MORDOR
}

\author{
Bilan de l'année 1994-1995
}

\author{
par R. Garçon
}

Electricité de France, Division Technique Générale, Service ressources en eau

\section{I - PRESENTATION DU MODELE HYDRO- LOGIQUE MORDOR}

MORDOR est un modèle qui simule le fonctionnement d'un bassin versant. Il a été développé en 1990 pour étendre le champ des méthodes utilisées par le Service Ressources en Eau pour le suivi et la prévision hydrologiques (fig. 1).

A travers MORDOR, un bassin versant est vu comme une réserve naturelle d'eau dont les variations expliquent l'écart entre le bilan atmosphérique (les précipitations moins l'évaporation) et le débit d'eau sortant du bassin par la rivière, que le modèle tente de calculer.

Le bassin versant est une entité physique éminemment complexe (il suffit d'imaginer, pour une goutte d'eau quittant

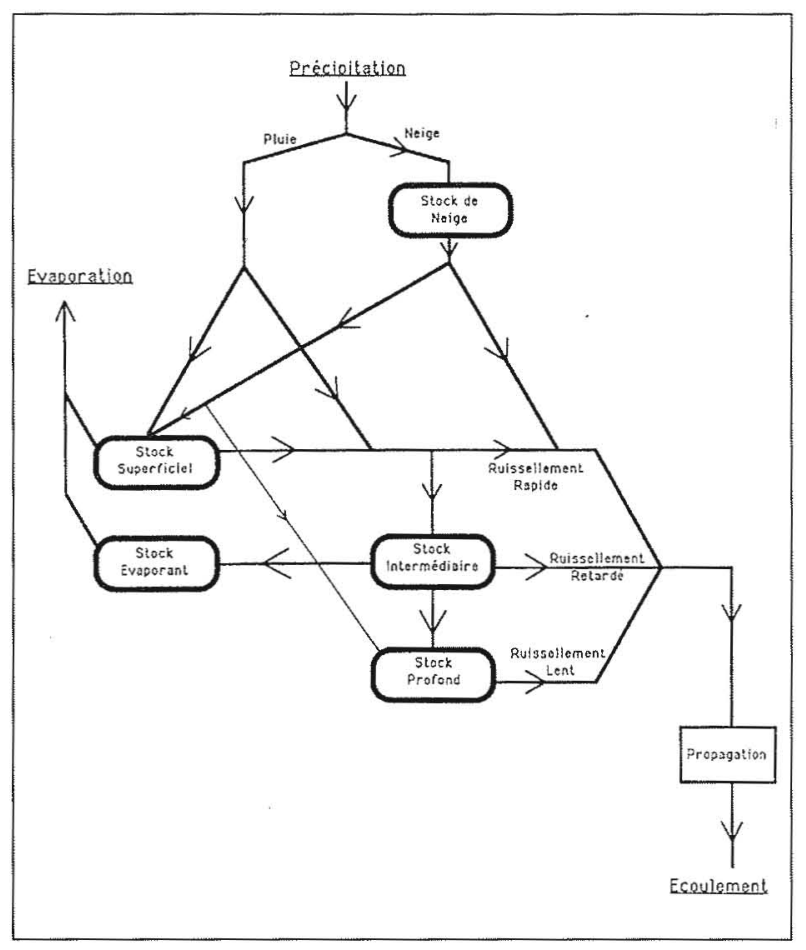

1. Schéma du modèle. le bassin par la rivière, le nombre d'itinéraires préalables possibles) que MORDOR schématise de façon radicale en un système de 5 réserves représentant chacune une forme différente de stockage de l'eau. Cette conceptualisation classe MORDOR parmi les modèles dits "à réservoirs". Un des stocks figure l'équivalent en eau du manteau neigeux tandis que les 4 autres réserves représentent l'eau retenue à la surface du bassin et à différents niveaux de profondeur.

MORDOR adjoint à cet ensemble les équations nécessaires à l'estimation des transferts entre ces cinq réserves, l'atmosphère et la rivière. Ces transferts sont eux-mêmes la schématisation de phénomènes physiques : précipitation, fonte, saturation, évaporation, infiltration, ruissellement, ..

L'hypothèse liée à une telle modélisation consiste à prétendre résumer tout le passé hydrologique du bassin étudié par le niveau de remplissage des différents réservoirs, véritables variables d'état du système. Un second pari consiste à vouloir appréhender l'essentiel de l'évolution de ces réserves à partir de deux données seulement, à savoir une précipitation spatiale et une température de l'air. Enfin MORDOR, quoique adaptable à d'autres pas de temps, a été plus particulièrement conçu en vue d'une utilisation au pas journalier.

Développé pour servir dans un cadre opérationnel, MORDOR marque sa différence vis-à-vis de modèles beaucoup plus sophistiqués, distribués dans l'espace, fonctionnant à un pas de temps plus fin et prenant explicitement en compte un nombre bien plus élevé de facteurs externes et d'éléments propres au bassin (vitesse du vent, rayonnement, humidité de l'air, structure du manteau neigeux, végétation, géologie, ...). On a, au contraire, privilégié la robustesse et la facilité de mise en œuvre puisque les données de précipitations et températures de l'air journalières sont les plus faciles à acquérir sur de longues périodes.

Au pas de temps journalier, MORDOR incrémente la valeur de son stock de neige en fonction des précipitations et de la température de l'air qui détermine la fraction des précipitations solides. La proportion de la surface enneigée, Gème variable d'état du modèle, est simultanément mise à jour. La fusion est ensuite estimée en fonction de cette proportion et de la température de l'air. Notons que, sur un bassin de montagne, on peut observer durant une même journée accumulation de neige en altitude et fusion dans les vallées. Le stock glaciaire est considéré comme infini à l'échelle de temps visée par le modèle. Contrairement aux autres stocks, les possibilités de contribution de ce réservoir à l'écoulement ne sont pas amoindries par ses contributions des jours précédents. 
Les précipitations liquides et l'eau de fonte se rejoignent alors dans la réserve (naturelle) de surface. Les processus d'infiltration et d'exfiltration entre les quatre réserves hydriques et le réseau hydrographique sont gérés à partir de paramètres réglant vidanges et débordements. L'évaporation - importante même en montagne — n'est pas négligée. Une fonction de transfert temporelle régit le cheminement de l'eau dans le réseau hydrographique jusqu'à l'exutoire du bas$\sin$.

Le schéma global du modèle est représenté figure 1. Les paramètres permettant au modèle de simuler l'écoulement sur un bassin donné sont, pour une partie d'entre eux, déduits des caractéristiques physiques du bassin et, pour l'autre partie, calés statistiquement à partir des données journalières de précipitations, de températures de l'air et de débits formant l'échantillon de calage.

\section{CALAGE DE MORDOR SUR LE BAS- SIN DE LA DURANCE}

Le barrage de Serre-Ponçon intercepte un bassin versant de $3720 \mathrm{~km}^{2}$ si l'on tient compte des débits dérivés du bassin de la Blanche. MORDOR a été calé sur ce bassin à partir des données des années 1961 à 1988. La précipitation spatiale a été calculée selon une combinaison linéaire des données de 8 postes pluviométriques, tandis que la température de l'air relevée à Embrun a été retenue comme principale référence thermique.
Pour la période de calage, les précipitations annuelles moyennes sont de $965 \mathrm{~mm}$ pour un écoulement de $700 \mathrm{~mm}$, l'évaporation estimée par le modèle expliquant la différence. Les débits simulés se rapprochent des débits observés de façon satisfaisante puisque les corrélations sont de 0,965 au pas journalier et de 0,984 au pas de temps mensuel.

Le modèle ainsi calé a ensuite été testé sur des années n'appartenant pas à l'échantillon de calage : on présente (fig. 2) graphiquement les résultats de la simulation par MORDOR de l'année hydrologique 1993-1994. Les irrégularités du débit observé sont liées au mode de calcul de cette variable (débits turbinés + variations de réserve, ce second terme étant sujet à des imprécisions se compensant dans le temps).

Le caractère nival de l'alimentation de la Durance (la moitié du bassin versant se situe au dessus de $2100 \mathrm{~m}$ d'altitude) se manifeste, pour l'année étudiée, par les apports élevés des mois de mai et juin et par les apports faibles de décembre à février malgré les précipitations soutenues de ces mois d'hiver. En mars, les débits remontent en deux temps, en dépit de l'absence de précipitations, sous l'effet de 2 vagues de douceur précoce alors qu'avril voit les débits redescendre malgré de nouvelles précipitations. Mais la fraîcheur d'avril explique que la fonte ne reprenne qu'en mai. Cette année - n'appartenant pas à l'échantillon de calage - est bien simulée par le modèle. Les imprécisions sur les premiers épisodes de fonte restent acceptables. L'écart entre débits simulés et observés en fin de période de fusion (juillet) montre les limites de Ia simulation. En volume, il représente moins de $1 \%$ des précipitations de l'hiver précédent. Une telle erreur, apparemment bénigne, ne devient

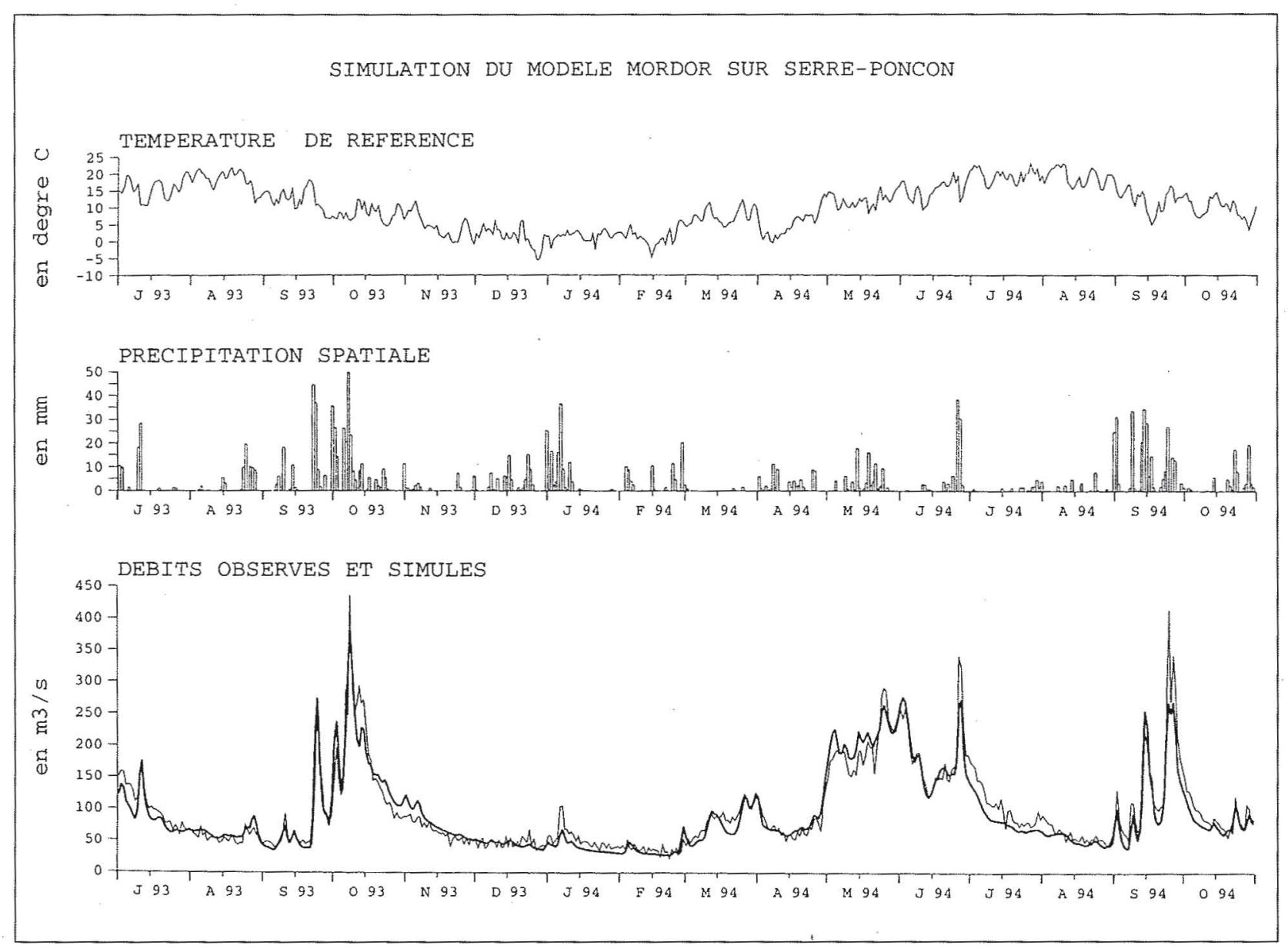

2. Simulation du modèle MORDOR sur Serre-Ponçon. 
vraiment décelable qu'en fin de fonte où elle se traduit alors par une erreur relativement importante. Cette figure, certai nement plus parlante qu'un froid coefficient de corrélation, montre à la fois les qualités du modèle et ses limites.

Globalement, ces tests ont constitué une garantie suffisante en vue d'une utilisation de MORDOR pour le suivi et la prévision des apports sur le bassin. On peut se satisfaire des résultats si l'on considère que les erreurs faites sur le débit sont équivalentes à celles qui résulteraient d'un biais de $1{ }^{\circ} \mathrm{C}$ dans la mesure de la température ou de $5 \%$ sur celle des précipitations (de tels biais ont été simulés artificiellement et on a constaté qu'ils doublaient la variance de l'erreur du modèle sur les volumes mensuels ou annuels). Cette observation amène deux remarques:

- La qualité des séries utilisées est prépondérante vis-à-vis des résultats du modèle.

- D'autre part, toute modélisation, même très sophistiquée, se heurterait à ce problème de qualité de mesure. Sans que cela doive être un frein aux progrès de la modélisation hydrologique et de la recherche dans ce domaine en général, on peut donc considérer que le degré de complexité de MORDOR est, aujourd'hui, tout à fait adapté à l'hydrologie opérationnelle et aux limites imposées dans ce cadre par des critères économiques.

\section{PRÉVISION DES APPORTS DE LA SAISON DE FONTE}

En année moyenne, la réserve en eau du bassin atteint son maximum en avril et son minimum en septembre. C'est évidemment le phénomène d'accumulation et de fusion du manteau neigeux qui est le principal responsable de cette variation saisonnière du stock. Les débits de mai à juillet correspondent, pour l'essentiel, à la baisse de cette réserve, quoiqu'ils puissent être également soutenus par des pluies de printemps et d'été.

Une bonne estimation de la quantité d'eau stockée permet donc une prévision des apports de printemps. Compte tenu de l'incertitude due aux précipitations futures, on ne peut donner en prévision qu'une "fourchette" ou, statistiquement parlant, un intervalle de confiance. Traditionnellement, cette prévision est élaborée à partir d'une combinaison linéaire d'un certain nombre de préviseurs. Les limites inhérentes à la régression nécessitent que ces préviseurs soient agrégés pour rester peu nombreux. On s'appuie, en priorité, sur le cumul des précipitations d'hiver ou sur le stock de neige mesuré à la date de la prévision. Mais l'influence des précipitations d'hiver sur les apports de printemps dépend de la fraction solide de ces précipitations, variable d'un hiver à l'autre, et difficile à apprécier globalement à partir d'une variable simple. Le manteau neigeux, quant à lui, présente deux inconvénients : il est irrégulier dans l'espace et donc difficile à évaluer. Par ailleurs, la réserve de neige, même si elle est prépondérante, n'est pas la seule réserve disponible.

Par rapport à de tels préviseurs, incomplets, les variables d'état calculées par MORDOR peuvent fournir une alternative intéressante : leur qualité réside dans leur faculté à synthétiser un grand nombre de variables élémentaires dont les influences sont croisées, non linéaires, et, par conséquent, insaisissables par régression brute.

La figure 3, par exemple, montre l'évolution de la réserve totale, cumul des cinq stocks de MORDOR, dont le stock de neige. Cette réserve est mesurée en millimètres de valeur en eau moyenne sur le bassin. Compte tenu de la surface drainée par la Durance, un millimètre d'eau représente ici 3,7 millions de $\mathrm{m}^{3}$.

On peut noter que cette réserve globale n'est jamais vide ce qui aurait comme conséquence le tarissement total de la Durance! Il résulte de cette remarque anodine qu'un stock

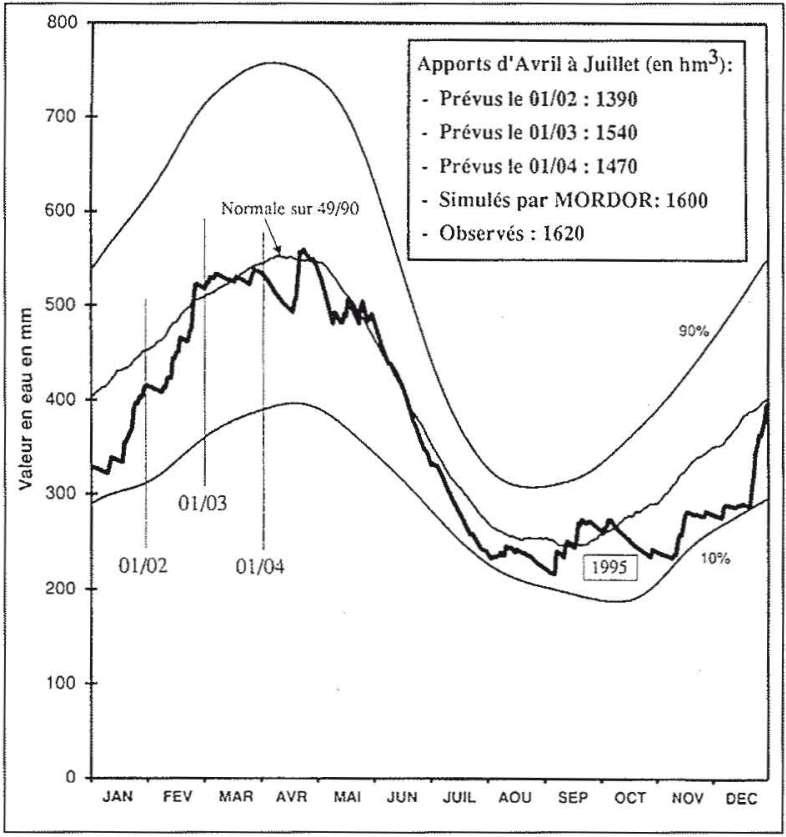

3. Modélisation du stock hydrique global en amont de la retenue de Serre-Ponçon. Modélisation par MORDOR de l'eau stockée dans le bassin sous toutes ses formes. Comparaison de la période récente aux statistiques 1949-1990.

de $500 \mathrm{~mm}$ ne garantit pas un écoulement correspondant au cours des mois suivants : d'une part, de l'eau retournera à l'atmosphère par évaporation et, d'autre part, les réserves ne se videront pas complètement. Par contre, les précipitations futures sont évidemment susceptibles de venir augmenter ces réserves.

Cette difficulté à interpréter directement les valeurs absolues des stocks nous ont amenés à porter sur le graphe les statistiques déduites de la simulation de MORDOR depuis 1949. Le niveau moyen des stocks calculés par le modèle à une date donnée est représenté par la courbe intermédiaire. Cette valeur est supérieure à la courbe médiane - non affichée ici - du fait de l'asymétrie positive de ces variablesstocks. Les deux courbes extrêmes repèrent l'évolution saisonnière des quantiles 10 et 90 .

L'année 1995 débute avec un stock déficitaire, proche du quantile 10 (le déficit du stock de neige est, par lui-même, encore plus important, mais le sous-sol du bassin garde le souvenir d'une crue en novembre 1994). Ce retard, à la date du ler janvier, est comblé par les précipitations supérieures à la normale des deux premiers mois de l'année. Par la suite, la réserve restera proche de la normale saisonnière malgré une petite baisse au mois de mars, en raison de faibles précipitations.

Compte tenu des rendements attendus de l'eau contenue dans les cinq. "réservoirs" de MORDOR, les prévisions des apports cumulés d'avril à juillet ont été de $1390 \mathrm{hm}^{3}$ au ler février, $1540 \mathrm{hm}^{3}$ au ler mars (soit les apports normaux) et $1470 \mathrm{hm}^{3}$ au 1er avril. Les pluies d'avril, juin et juillet ayant été excédentaires, les apports simulés par le modèle ont finalement été de $1600 \mathrm{hm}^{3}$ pour un écoulement réel de 1620 $\mathrm{hm}^{3}$. Les "fourchettes" émises aux différentes dates ont toutes encadré cette valeur.

\section{PREVISIONS A COURT TERME}

MORDOR a également été utilisé dans le cadre de la prévision à court terme $(\mathrm{J}$ à $\mathrm{J}+4)$ des débits entrant dans la rete- 
nue. Le principe de cette prévision est, en théorie, assez simple: il suffit de prolonger les séries de précipitations et températures observées par les valeurs prévues pour les jours suivants, pour que le modèle soit à même de proposer une simulation de l'évolution future des débits. Mais deux obstacles viennent affecter la précision d'une telle mécanique En premier lieu, la prévision météorologique est elle-même entachée d'incertitudes importantes.

\subsection{Contrôle de la qualité des prévisions météorolo- giques}

Les prévisions à court terme du débit de la Durance ont été remises à jour 2 à 3 fois par semaine pendant la période expérimentale, d'octobre 1994 à octobre 1995, par l'équipe du Centre hydrométéorologique Alpes (S. Caron, M.F. Compagne, D. Parrel, R. Garçon). On dispose donc d'un peu plus de 100 prévisions pour dresser ce bilan.

Le matin, le prévisionniste sélectionne le scénario moyen le plus vraisemblable à ses yeux pour l'évolution future des températures de l'air et des précipitations. Il est aidé en cela par les prévisions de Météo France (modèle ARPEGE et adaption statistique) qu'il interprète en fonction de son expérience. Compte tenu de ce travail, le contrôle ci-dessous vise simplement à apprécier la performance globale de la chaine de prévision et à identifier les points où l'on a encore à progresser sans remettre en cause les outils de Météo France dont on ne saurait se passer. La température de l'air utilisée dans le cadre de la prévision opérationnelle est la température journalière (moyenne des températures minimale et maximale du jour) de la station d'Embrun. Le tableau $I$ donne les corrélations entre les variations prévues et observées de cette température à partir du jour $\mathrm{J}-1$ :

Ces résultats sont corrects mais cachent quelques erreurs de prévision supérieures à $4^{\circ} \mathrm{C}$ qui peuvent causer une mauvaise appréciation du débit de fonte ou même une grave erreur sur les conséquences immédiates d'un passage perturbé (mauvaise estimation de la limite pluie-neige).

Un contrôle identique a été effectué sur les prévisions de la précipitation spatiale journalière (cumulée de $8 \mathrm{~h}$ à $8 \mathrm{~h}$ ) dont on voit les résultats sur le tableau 2.

L'évolution des corrélations montre, comme on pouvait s'y attendre, une dégradation sensible de la qualité des prévisions avec l'éloignement de l'échéance. Des erreurs de $15 \mathrm{~mm}$ sont parfois commises pour les précipitations des 24 prochaines heures et, à partir de $J+2$, on observe des écarts supérieurs à $20 \mathrm{~mm}$.

De telles erreurs de prévision sont évidemment très pénalisantes pour les prévisions hydrologiques. C'est bien sur ce maillon que les plus gros efforts doivent porter même si cet exercice est intrinsèquement difficile, notamment au delà de $\mathrm{J}+2$.

\subsection{Contrôle des prévisions de débits}

En dehors des problèmes liés aux prévisions météorologiques, un deuxième obstacle réside dans la simulation hydrologique elle-même. Les imperfections de la modélisation peuvent entraîner un écart entre débits simulés et observés. En l'absence d'un tel écart au moment de la prévision, on peut considérer que le calage du modèle a atteint son objectif et que tout incite à accepter telles quelles les projections hydrologiques de MORDOR dans le futur. Par contre, des erreurs de reconstitution des débits récents peuvent signaler le risque quiune imperfection de la modélisation ou des données du passé continue à biaiser systématiquement les résultats du modèle pendant une certaine période.

La réduction automatique de ce biais fait l'objet de nombreux travaux menés au Service Ressources en Eau sous le titre générique de "recalage" (D. Gouy, Y. Gregoris, C. Dufour). Ce problème est néanmoins difficile à résoudre dans le cas général, les avancées les plus significatives ayant été accomplies dans le domaine des débits d'étiage.

Dans le cadre de la présente expérimentation, le "recalage" a été entièrement confié au prévisionniste qui interprète les écarts entre observations et simulations passées et corrige en conséquence les prévisions élaborées par MORDOR. Ces prévisions montrent quelques erreurs importantes, dont la plus spectaculaire est la sous-estimation au jour J de la crue du 24 septembre 1994 (problème de prévision de précipitation), mais aussi quelques réussites, notamment durant la période de fusion (fig. 4).

Globalement, les résultats sont satisfaisants comme en témoignent les corrélations des débits prévus et simulés calculées en variation par rapport au dernier débit connu $(\mathrm{J}-1)$. Il faut noter qu'au moment d'établir sa prévision, le prévisionniste est déjà informé des débits de la Durance et de l'Ubaye à l'amont immédiat de la retenue pour les premières heures du jour J (tableau 3).

Tableau 1. - Qualité des prévisions de température du CHA. Corrélations des variations observées et prévues à partir de $\mathrm{J}-1$.

\begin{tabular}{|l|c|c|c|c|c|}
\hline ECHEANCE & $\mathrm{J}$ & $\mathrm{J}+1$ & $\mathrm{~J}+2$ & $\mathrm{~J}+3$ & $\mathrm{~J}+4$ \\
\hline CORRELATION & 0,72 & 0,75 & 0,79 & 0,73 & 0,73 \\
\hline
\end{tabular}

Tableau 2. - Qualité des prévisions de précipitation du CHA. Corrélations des cumuls $8 \mathrm{H}-8 \mathrm{H}$ observés et prévus.

\begin{tabular}{|l|c|c|c|c|c|}
\hline ECHEANCE & $\mathrm{J}$ & $\mathrm{J}+1$ & $\mathrm{~J}+2$ & $\mathrm{~J}+3$ & $\mathrm{~J}+4$ \\
\hline CORRELATION & 0,72 & 0,64 & 0,53 & 0,26 & 0,33 \\
\hline
\end{tabular}


Tableau 3. - Qualité des prévisions de débit de la Durance à Serre-Ponçon. Corrélations des variations observées et prévues à partir de J-1.

\begin{tabular}{|l|c|c|c|c|c|}
\hline ECHEANCE & $\mathrm{J}$ & $\mathrm{J}+1$ & $\mathrm{~J}+2$ & $\mathrm{~J}+3$ & $\mathrm{~J}+4$ \\
\hline CORRELATION & 0,80 & 0,74 & 0,70 & 0,86 & $0,80^{*}$ \\
\hline
\end{tabular}

* Compte tenu de la difficulté à prévoir, 4 jours à l'avance, les précipitations et températures (cf 4/1), la prévision à J+4 n'a pas été envoyée systématiquement et la corrélation est calculée sur 35 prévisions seulement.

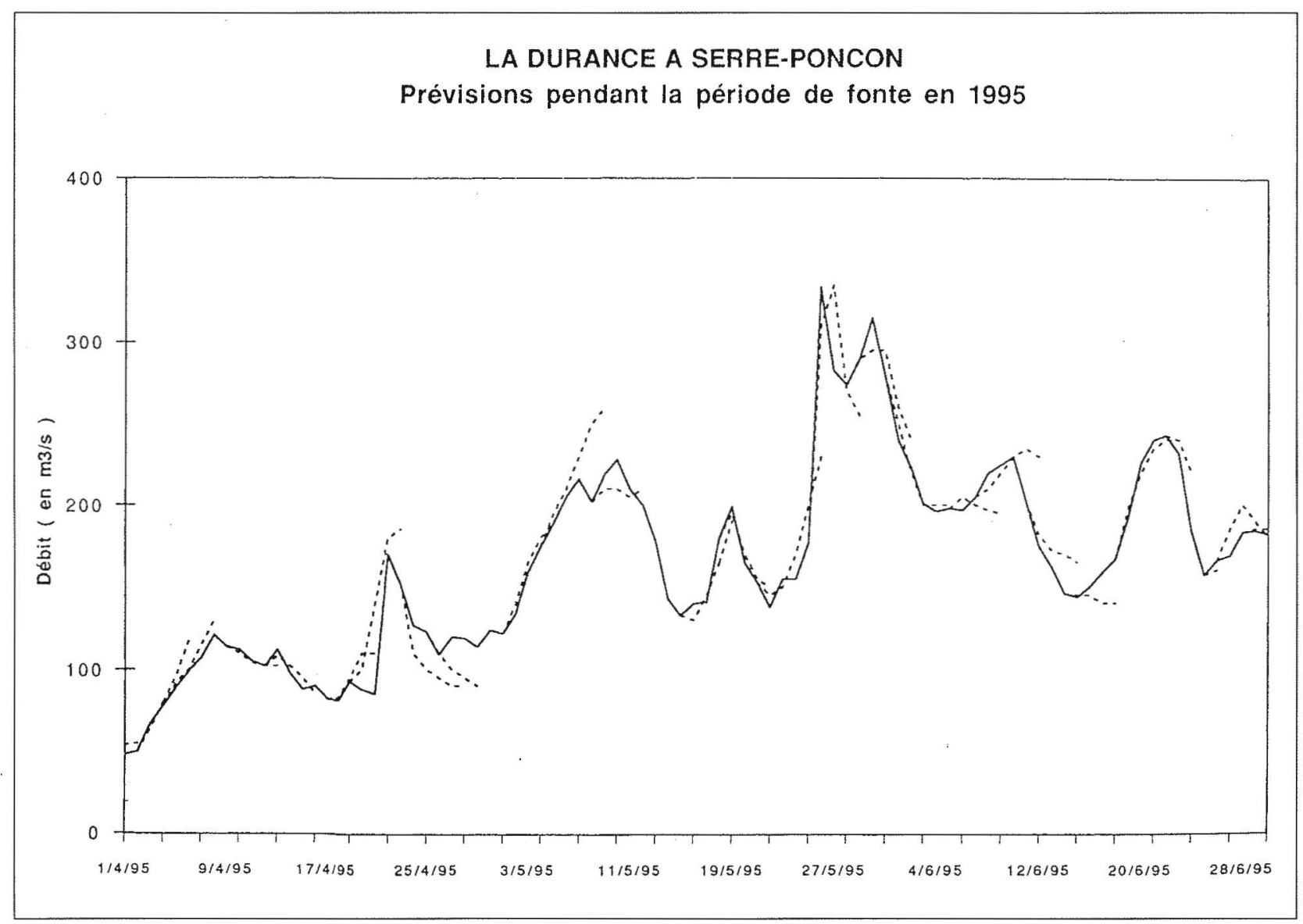

4. La Durance à Serre-Ponçon. Prévisions pendant la période de fonte en 1995.

Pour évaluer la qualité intrinsèque du modèle en dehors de tout problème de prévision météorologique, on a, a posteriori, calculé les corrélations entre variations de débits observées et simulées par MORDOR avec les précipitations et températures réelles. Ces simulations de prévision en "futur connu" ne bénéficient pas, par contre, de l'expertise de l'hydrologue. Ces nouvelles corrélations sont calculées sur un nombre de jours 3 à 4 fois plus élevé puisqu'on a pu utiliser tous les jours de l'année (tableau 4).

Comme on pouvait s'y attendre, ces corrélations sont, en moyenne, meilleures que celles relatives aux prévisions opérationnelles, mais l'écart n'est pas si important en regard des erreurs de prévisions météorologiques, ce qui révèle peutêtre - l'échantillon est un peu petit pour qu'on puisse se prononcer catégoriquement - l'expertise hydrologique des prévisionnistes.

\section{$\mathrm{V}$ 圆 CONCLUSION}

Cette première expérimentation de MORDOR dans le cadre de la prévision hydrologique opérationnelle est intéressante à plusieurs titres.

Le support de la modélisation a aidé le prévisionniste à passer de la prévision qualitative à la prévision quantitative sur un bassin où seules les prévisions d'apport à long terme étaient quantifiées jusqu'à présent. Le franchissement de ce cap est important en termes de service rendu à l'exploitant et il permet par ailleurs une appréciation objective de la qualité des prévisions.

Le suivi de l'état du bassin versant par l'hydrologue a incontestablement progressé, grâce aux synthèses fournies par les stocks du modèle qui viennent en complément des moyens d'investigation classiques (nivométrie par exemple). 
Tableau 4. - Qualité des prévisions de débit de la Durance à Serre-Ponçon,. Corrélation des variations observées et "prévues" à partir de $\mathrm{J}-1$.

\begin{tabular}{|l|c|c|c|c|c|}
\hline ECHEANCE & $\mathrm{J}$ & $\mathrm{J}+1$ & $\mathrm{~J}+2$ & $\mathrm{~J}+3$ & $\mathrm{~J}+4$ \\
\hline $\begin{array}{l}\text { PREVISIONNISTE } \\
\text { EN TEMPS REEL }\end{array}$ & 0,80 & 0,74 & 0,70 & 0,86 & 0,80 \\
\hline $\begin{array}{l}\text { MODELE SEUL EN } \\
\text { FUTUR CONNU }\end{array}$ & 0,75 & 0,81 & 0,83 & 0,84 & 0,85 \\
\hline
\end{tabular}

Le rendement des précipitations à court, moyen et long termes et l'effet de l'évaporation reçoivent une explication qui, à défaut d'être parfaite, procure une base pédagogique à l'hydrologue, l'invitant à rationaliser son expérience.

Enfin, les résultats sont très encourangeants. Les corrélations entre variations prévues et observées du débit atteignent des valeurs de 0,75 à 0,80 qui incitent, en elles-mêmes, à généraliser cette démarche à la fois aux bas- sins amont des réservoirs importants pour l'exploitant et aux bassins intermédiaires menaçant régulièrement d'encombrement les vallées à l'aval de ces retenues.

La qualité de ces résultats n'est, bien sûr, pas le fait de la seule modélisation mais repose également, d'une part, sur des mesures hydroclimatologiques de qualité - effectuées pendant une période suffisante pour le calage du modèle et, d'autre part, sur des prévisions météorologiques qui profitent du travail accompli par Météo France. 